\title{
Discussion on Common Treatment of Thrombosis Angiitis Obliterans
}

\author{
Yanan $\mathrm{Li}^{1,2, a}$, Xiaona Guo, b and Chunsheng $\mathrm{Yan}^{3, \mathrm{c}^{*}}$ \\ ${ }^{1}$ Henan Province Hospital of TCM, 450000, Henan, P. R. China \\ ${ }^{2}$ The Second Affiliated Hospital of Henan University of Traditional Chinese Medicine, 450000, Henan, \\ P. R. China \\ ${ }^{3}$ Huanghe Science and Technology College, Zhengzhou, 450003, Henan, P. R. China \\ azwxglyn@126.com, ${ }^{\mathrm{a}}$ Ig8509840@163.com, ${ }^{\mathrm{c} * 331318321 @ q q . c o m}$ \\ ${ }^{*}$ The corresponding author
}

Keywords: Thrombosis angiitis obliterans (TAO); Diagnosis method; Treatment method; Clinical treatment

\begin{abstract}
Thrombosis angiitis obliterans (TAO) is a common chronic peripheral occlusive vascular inflammatory lesions. Symptoms appear in the limbs, the small arteries and veins, but the most common in the lower extremities, may also affect the mesentery, cerebrovascular and coronary arteries. This paper analyzes the the causes and clinical manifestations of thrombosis obliterans, several common clinical treatment of thrombosis obliterans summarized. Although the treatment of thrombosis obliterans varied, but whether it is drugs or surgery, so far still no very good effect, and can treat all patients received. Through a comprehensive analysis of the commonly used method of mass thrombosis obliterans for clinical treatment provides some suggestions.
\end{abstract}

\section{Introduction}

Thrombosis angiitis obliterans, (TAO) is a non-atherosclerotic inflammatory disease of the intercept, the pervasive influence of small arteriovenous limbs. In the acute phase there is less occlusion and thrombosis. It tends to young men. The main factors to consider its current tobacco consumption, a rare systemic symptoms and signs of thrombosis and hypercoagulability relative ischemia of the limbs, acute phase reactants and no specific immune markers. So far, the cause of the TAO is not yet entirely clear, but the literature shows that tobacco use and exposure plays an important role in the development of diseases. While genetic factors and hypercoagulable state, endothelial dysfunction, infection factors and immune mechanisms, etc. are the occurrence of this disease-related, and development impact and incidence of this disease is most closely on tobacco exposure. As shown in Fig. 1.
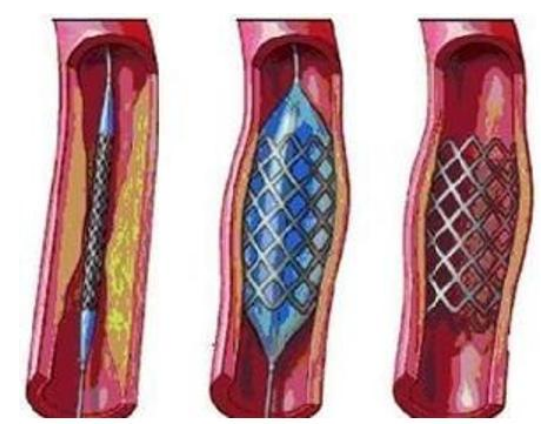

Figure 1. The formation of TAO

Pathological characteristics Thrombosis angiitis obliterans is divided into three phases, the first stage is the acute phase of the lesion phase, which is characterized by acute inflammation exhibits covering all of the outer peripheral wall of the blood vessel, especially vein, while occlusion thrombosis. The outer periphery of the thrombus, how often polymorphonuclear leukocytes and 
nuclear rupture, so-called micro-abscesses. The first stage is an intermediate stage, intermediate stage in occlusive arterial and venous thrombosis. At this stage, there is usually prominent infiltration of inflammatory cells in thrombosis, more or less infiltration colleagues around the vessel wall. The third stage is the chronic phase, the phase or end-stage chronic disease characterized by occlusive thrombus organization and extensive revascularization, major blood vessels become fibrosis media and perivascular. In all three phases, the organizational structure of the occlusive thrombus normal, including endometrial elasticity still intact. These findings can be correctly differentiate patients with atherosclerosis and other vascular inflammation. Because there is a change significantly intimal damage and age disproportionate membrane in these patients, Thrombosis angiitis obliterans is a segmental distribution, was "jumping" Distribution.

Thrombosis angiitis obliterans the cause is still unclear, generally considered the following factors [1]:

Smoking. Comprehensive domestic and international data, Thrombosis angiitis obliterans patients, smokers accounted for $60 \%$ to $95 \%$. Clinical observations, quitting smoking can make Thrombosis angiitis obliterans disease remission, but also make smoking again deteriorated. Erb and other animal experiments in mice found that tobacco leachate can cause vascular disease. Harkavy etc. for tobacco leachate intradermal test found, Thrombosis angiitis obliterans patients with positive rate of 78 to $87 \%$, while only 16 to $46 \%$ of normal. But after all, still the minority, part of Thrombosis angiitis obliterans patients Thrombosis angiitis obliterans occurs smokers and had no history of smoking. Thus, smoking may be an important factor in the pathogenesis of Thrombosis angiitis obliterans, but not the only cause.

Cold, Wet, Trauma. incidence of Thrombosis angiitis obliterans of relatively cold northern high. Epidemiological survey found that 80 percent of Thrombosis angiitis obliterans patients before the onset of exposure to cold and damp history; some patients have a history of trauma. These factors may cause vasospasm and vascular endothelial injury, and lead to vascular inflammation and thrombotic occlusion.

Infections, Malnutrition. clinical observations, many Thrombosis angiitis obliterans patients have a history of recurrent fungal infections. Thompson found hair streptozotocin skin test positive rate Thrombosis angiitis obliterans patients with $80 \%$, while the control group only $20 \%$. Craven believes that the body's immune response to fungi, induce increased blood fibrinogen and hypercoagulable state may be related to the pathogenesis of Thrombosis angiitis obliterans. Researchers found that in many countries, Thrombosis angiitis obliterans more common in low income and living standards of the population. After Hill and other analysis of Indonesia's Thrombosis angiitis obliterans found that most patients lack of protein in the diet, especially the essential amino acids. There are people in making rat study found that diet and lack VitB1 VitC can be induced in rats vasculitis. Thus, protein, vitamin B1 and vitamin $\mathrm{C}$ deficiency may be related to the disease.

Hormonal Disorders. Thrombosis angiitis obliterans vast majority of patients were male (80 to $90 \%$ ), and both times the incidence in young adults. Some people think that prostate disorder or excessive loss of prostatic fluid, the body can have a dilation of blood vessels and inhibit platelet aggregation reducing prostaglandins, and has the potential to peripheral vasomotor dysfunction, thrombosis leading to the disease.

Genetic. Thrombosis angiitis obliterans patients, 1 to $5 \%$ have a family history. Many scholars have found that human leukocyte antigen (HLA) loci and some special Thrombosis angiitis obliterans related to the pathogenesis. Japanese researchers found that HLA-J-1-1 Thrombosis angiitis obliterans positive rate of $46 \%$, while only $18 \%$ of normal. Another report, Thrombosis angiitis obliterans in patients with HLA-BW54, HLA-BW52 and HLA-A positive rate increased. Wherein the HLA-J and HLA-BW54 governed by genetic factors dominate.

Neurovascular Dysregulation. the autonomic nervous system stimulation of endogenous or exogenous adjustment disorders, blood vessels easily spasticity. Vasospasm can make long-term damage to the wall, hypertrophy clots lead to vascular occlusion. 
An Autoimmune Disorder. almost 10 years, autoimmune factors in the pathogenesis of Thrombosis angiitis obliterans role and more attention. Gulati et al found Thrombosis angiitis serum obliterans in IgG, IgA, and IgM increased significantly, while the complement $\mathrm{CH} 50$ and $\mathrm{C} 3$ decreased, and found that anti-artery antibodies in the blood vessels of patients with serum and lesions and arteries have a strong affinity for immune complexes thereof. Smoler and other collagen antibody found seven cases in 20 cases Thrombosis angiitis obliterans, whereas the control group without the presence of one case of collagen antibody. Bollinger and so on and so on, respectively Berlit elastin antibody found in Thrombosis angiitis obliterans in. Gulati and so that, smoking and other factors could alter vascular antigen, produce its own antibodies anti artery. Thus formed immune complex deposition in the patient's blood vessels leading to inflammation and thrombosis.

\section{Clinical Manifestations and Diagnosis}

Thrombosis angiitis obliterans patients with the slow progress of the disease, often a chronic, after a long period of evolution, the condition was gradually increased. The clinical manifestations mainly due to decreased blood flow after arterial occlusion caused by limb ischemia. The severity is based on the site of vascular obstruction. The scope and extent of collateral circulation and the local availability of secondary infection and other conditions vary. Common symptoms include cold and paresthesia, intermittent claudication, pain, rest pain, paresthesia, skin color changes, arterial pulse weakened or disappeared, nutritional disorders, migratory superficial phlebitis thrombosis.

Coldness and paresthesia: cold limbs, cold is common early symptoms. Affected surface temperature decreases, especially in the toe (finger) end the most obvious. Due to ischemic nerve endings affected by the limb (toe, finger) there may be a sense of the corpus callosum, tingling, numbness or burning and other sensory abnormalities.

Pain: Early symptoms also originated in arterial spasm, due to nerve endings feel the blood vessel wall and surrounding tissues and organs caused by irritation, pain usually is not severe.

Intermittent claudication: it was due to meningitis and thrombosis and arterial occlusion produced a special manifestation of ischemic pain. That is when the patient after walking some distance, leg or foot muscles ache or pain occurs, if it continues to walk, the pain worsened and had to stop after a short break, immediately relieve pain, pain and re-appear again after the walk, which kinds of symptoms of intermittent claudication. With the progress, gradually shorten the walking distance, stop the growth of the rest of the time.

Rest pain: the disease continues to develop, more severe arterial ischemia, pain, severe and persistent, even when the body is at rest, the pain is still more than called rest pain. Worse at night, limb elevation increase, after sagging a little pain can be reduced. Patient enough to sit day and ask knees, overnight. Sometimes drooping limb bedside, to relieve pain, if complicated by infection, pain is more intense.

Skin color changes: due to ischemia caused skin pale, with superficial vascular tone weakened by thinning of the skin, can still occur flushing or cyanosis.

Artery pulse weakened or disappeared: artery, ulnar or radial artery pulse after dorsal or tibia, with the lesion progression weakened and even disappear.

Nutrition disorders: chronic limb ischemia, tissue dystrophy, manifested as dry skin, scaling, chapped, hair loss, toe (finger) A thickening, deformation and slow growth, the calf muscle relaxation, atrophy, circumference change fine. Deterioration of the disease, severe limb ischemia, and finally to produce ulcers or gangrene [2]. Mostly dry gangrene, first appear in the next one or two of the end of the toe or toenail, then involving the entire toe. At the beginning of the dry toe black, necrotic tissue formed after prolonged healing of ulcers. At this time more severe limb pain, day and night, the patient can not sleep, appetite reduction, weight loss, weakness, pale greenish yellow and even anemia. If the concurrent infection, gangrene was wet, then high fever, chills, irritability and other symptoms of sepsis. 
Migratory superficial vein thrombosis inflammation: about $1 / 2$ of the patients before the onset or the onset of the process, superficial vein in the leg or foot, recurrent thrombophlebitis migratory superficial phlebitis. Showed superficial venous involvement red cord, nodular, with mild pain, acute episodes for 2 to 3 weeks after symptoms subside, then be repeated over a period of time, since the disease for months or years without being patient note.

\section{Common Treatment to Analyse}

Common treatment methods can be divided into drug treatment and surgery.

Medical Treatement. (1) Smoking is definitely an important measure to prevent and treat this disease. (2) feet clean and dry to keep feet clean and prevent infection; wet than dry and cold because the disease is more harmful, it should keep the feet dry; section has been suffering from poor blood circulation, even minor trauma can lead to tissue necrosis and also ulcer formation, it should not have any kind of trauma [3]. (3) weatherization whether at work or rest are advised to keep feet warm, to improve blood circulation foot, but can not overheat, so as not to increase the oxygen consumption. (4) changes in posture and foot movement of labor should change position at any time, in order to facilitate circulation. Usually available for foot motion (Buerger movement) to promote limb collateral circulation. Method: the patient supine, Raise wounded limb $45^{\circ}$, maintain $1 \sim 2 \mathrm{~min}$, then two foot drop bedside $2 \sim 5 \mathrm{~min}$, while two feet and toes to the surrounding activities 10 times, then the flat limb rest $2 \mathrm{~min}$, so repeated exercise five times per day back. (5) avoid the use of vasoconstrictor drugs.

Surgical Treatment. Staging arteriovenous bypass, arteriovenous bypass staging, also known as venous artery, and its basic principle is that by doing so thin tube, the arterial blood supply along the vein distal to improve the nutritional status of ischemic tissue. Stage arteriovenous shunt model of artery - vein - vein [4]. It is a new special blood circulation system. In order to be able to follow the blood veins have one-way valves open countercurrent flow in the ischemic tissue. Arterial vein, multi autologous vein bypass, a small number of artificial blood vessels. The length and diameter artificial blood vessels is not limited, but its high cost and easy to form clots. Autogenous vein compatibility and better compliance, and joint activities is not restricted. Patency bypass surgery is a major problem. According to the literature. Autologous vein as arterial substitute Recent patency rate of $80 \%-90 \%$, after about $2.5 \%$ annual rate of decline, long-term patency rate is still not ideal. Postoperative complications such as leg varicose veins, skin ulcers, heart failure, limb pain and other. Because arteriovenous blood flow is non-physiological, clinical applications require strict compliance with indications. The procedure is the following selectable: (1) extensive arterial occlusion, and intermittent claudication, rest pain, limb gangrene. (2) no distal arterial outflow tract, unable arterial bypass surgery. (3) conservative medical treatment more than a year and no significant improvement in symptoms. (4) additional tests are deep vein patency. (5) facing the risk of amputation.

Chemical inactivation technique sympathetic sense of sexual intercourse, as early as 80 years ago it was the use of lumbar sympathectomy treatment of lower extremity arterial ischemic disease, the principle is the removal of lumbar sympathetic ganglia can make lower limb arteries dominated sympathetic generated loss effects of dilation of the blood vessels, thereby increasing blood flow in the limbs, improve blood circulation.

The greater omentum, omentum which is rich in blood vessels, this surgery is to improve the blood supply to the ischemic area by means of omental transplantation, use one of the blood vessels. The principle is based on the distribution of large retinal blood vessels to maintain normal blood circulation under its cut, trimmed strips leads to the abdominal cavity, transferred to a limb calf, and placed under the deep fascia. Because of the greater omentum with extensive arterial and venous blood and lymph vessels, transplant easily establish collateral circulation and the surrounding tissue, so that the blood supply of ischemic limbs get. Omental transplantation can delay or even avoid amputation.

Percutaneous transluminal angioplasty, the basic method is through a pressurized balloon compression of atherosclerotic plaque and artery wall, so that the lumen to expand, maintain the expanded state of the lumen of the artery pulse. In recent years, with the development of interventional 
techniques and materials, PTA to trauma, repeatability and other advantages, as below-knee arterial stenosis or occlusion of the preferred method of treatment, particularly Deep balloon invention for the treatment of ischemic diseases knee belt He came new hope.

Lumbar sympathectomy surgery (LS) lumbar sympathectomy treatment TAO has been very controversial in clinical practice, many experts and scholars believe that lumbar sympathectomy can relieve arterial spasm TAO patients, foreign scholars reported this method can be lifted limb pain, and improve microcirculation organizations, and domestic think this method for improving skin blood flow good results for deep muscle tissue to improve blood flow is poor, the efficacy of intermittent claudication little, its long-term effects are yet to be verified.

Epidural spinal cord stimulation (SCS) This technique is carried out recently by the epidural implanted electrical stimulator, which can effectively relieve pain. Improve limb blood circulation and promote ulcer healing.

Interventional therapy, interventional therapy is divided into a simple expansion of the narrow blood vessels known as balloon angioplasty and stent placement after expansion that is stenting, interventional therapy.

\section{Conclusion}

Thrombosis obliterans treatment, although many different ways, but the drug therapy and surgery, so far still no effect and can be very good treatment for all patients received. This paper analyzes and summarizes the various treatment methods currently used TAO to provide more ideas for doctors and patients.

\section{References}

[1] Wang Jiaju, Zhao Wenguang,Sun Siqiao etc. TAO incidence Changes (One Hundred Years Review) [J]. Chinese extravascular. 2009, 2 (1): 7-9.

[2] Xu xin, Yang niu Fu Weiguo. Endovascular treatment of Thrombosis angiitis obliterans [J]. Chinese Journal of General Surgery, 2009, 24: 463-465.

[3] Ge Xiaohu, Sai Limu, Li Xuesong. Arteriotomy embolectomy treatment Thrombosis angiitis obliterans [J]. Chinese Journal of Bases and Clinics in 2010, 17: 672-675.

[4] wang mingyi, wang feng, ji donghua. Interventional treatment of Thrombosis angiitis obliterans of lower limb [J]. Interventional Radiology, 2012, 21 (10); 850-854. 